Article

\title{
Casimir Friction between Dense Polarizable Media
}

Johan S. Høye ${ }^{1}$ and Iver Brevik ${ }^{2, *}$

${ }^{1}$ Department of Physics, Norwegian University of Science and Technology, Trondheim N-7491, Norway; E-Mail: johan.hoye@ntnu.no

${ }^{2}$ Department of Energy and Process Engineering, Norwegian University of Science and Technology, Trondheim N-7491, Norway

* Author to whom correspondence should be addressed; E-Mail iver.h.brevik@ntnu.no; Tel.: +47-7359-3555; Fax: +47-7359-3491.

Received: 28 June 2013, in revised form: 17 July 2013 / Accepted: 22 July 2013 /

Published: 29 July 2013

\begin{abstract}
The present paper-a continuation of our recent series of papers on Casimir friction for a pair of particles at low relative particle velocity-extends the analysis, so as to include dense media. The situation becomes, in this case, more complex, due to induced dipolar correlations, both within planes and between planes. We show that the structure of the problem can be simplified by regarding the two half-planes as a generalized version of a pair of particles. It turns out that macroscopic parameters, such as permittivity, suffice to describe the friction, also in the finite density case. The expression for the friction force per unit surface area becomes mathematically well-defined and finite at finite temperature. We give numerical estimates and compare them with those obtained earlier by Pendry (1997) and by Volokitin and Persson (2007). We also show in an appendix how the statistical methods that we are using correspond to the field theoretical methods more commonly in use.
\end{abstract}

Keywords: Casimir friction; Casimir effect; van der Waals friction

\section{Introduction}

The typical situation envisaged in connection with Casimir friction is the one where two parallel semi-infinite dielectric nonmagnetic plates at micron or semi-micron separation are moving longitudinally with respect to each other, one plate being at rest, the other having a nonrelativistic velocity, v. Usually the plates are taken to have the same composition, their permittivity, $\varepsilon(\omega)$, being frequency-dependent. 
Most previous works on Casimir friction are formulated within the framework of macroscopic electrodynamics. Some references in this direction are [1-14]. In particular, the application of the theory to graphene materials is a very promising avenue of approach; $c f$., for instance, [9]. In the present paper, we focus on the following themes:

- We make use of statistical mechanical methods for harmonic oscillators, moving with respect to each other with constant velocity, $\mathbf{v}$, at a finite temperature, $T$. We claim that such a strategy, formally perhaps simpler than field-theoretical methods, is actually quite powerful. We have used this method repeatedly in previous recent investigations [15-19]; $f f$., also the earlier papers [20,21] in which the foundations of the method were spelled out. The essence of the method is to generalize the statistical mechanical Kubo formalism to time-dependent cases.

- These methods are then used to generalize the theory to the case of dense media. This is a nontrivial task, as the additivity property holding for dilute media is no longer valid. This topic is dealt with from Section 4 onwards. One will have to deal with a more complicated form of the Green function. The atomic polarizabilities appearing in the theory of dilute media have to be replaced by functions based upon the frequency-dependent permittivity. A noteworthy property is, however, that the permittivity, i.e., a macroscopic quantity, suffices to express the Casimir friction, even in the case of finite densities.

- It turns out that the friction force becomes finite at finite temperature, although usually small. As a numerical example, treated in Section 5, we find for the case of a gold metal that the force per unit surface area for equal plates at room temperature at small separation $(10 \mathrm{~nm})$ and moderate relative velocity $(100 \mathrm{~m} / \mathrm{s})$ becomes of the order, $10^{-11} \mathrm{~Pa}$. This, of course, cannot be measured. However, by changing input parameters, this will change rapidly, so that $F$ can become large. The situation is very sensitive with respect to input parameters. We make numerical comparisons with earlier works, notably Pendry (1997), and Volokitin and Persson (2007).

- As it is of interest to trace out the connection with the more standard field theoretical methods, we focus on this subject in Appendix B. Appendix A shows or indicates the formal background for the correlation functions used.

We mention that the microscopic approach has been followed by other investigators also, especially by Barton [22-24]. The equivalence between our approaches, actually a rather a nontrivial correspondence, has been shown by us explicitly [17].

For reasons of readability, we begin in the next section by summarizing essential points of the theory for dilute media [18,19]. Then, after giving an account of Fourier methods in Section 3, we embark, as mentioned, on the general case of finite particle density in Section 4.

\section{Dilute Media}

For a pair of polarizable particles, the electrostatic dipole-dipole pair interaction perturbs the Hamiltonian by an amount:

$$
-A F(t)=\psi_{i j} s_{1 i} s_{2 j}
$$


where the summation convention for repeated indices, $i$ and $j$, is implied. $s_{1 i}$ and $s_{2 j}$ are components of the fluctuation dipole moments of the two particles $(i, j=1,2,3)$. With electrostatic dipole-dipole interaction, we can write:

$$
\psi_{i j}=-\frac{\partial^{2}}{\partial x_{i} \partial x_{j}} \psi, \quad \psi=\frac{1}{r}
$$

(i.e., $\left.\psi_{i j}=-\left(3 x_{i} x_{j} / r^{5}-\delta_{i j} / r^{3}\right)\right)$. Here, $\mathbf{r}=\mathbf{r}(t)$ with components $x_{i}=x_{i}(t)$ is the separation between the particles. The time dependence in Equation (1) is due to the variation of $\mathbf{r}$ with time, $t$, and the interaction will vary as:

$$
-A F(t)=\left[\psi_{i j}\left(\mathbf{r}_{0}\right)+\left(\frac{\partial}{\partial x_{l}} \psi_{i j}\left(\mathbf{r}_{0}\right)\right) v_{l} t+\ldots\right] s_{1 i} s_{2 j}
$$

where $v_{l}$ are the components of the relative velocity, $\mathbf{v}$. The components of the force, $\mathbf{B}$, between the oscillators are:

$$
B_{l}=-T_{l i j} s_{1 i} s_{2 j}, \quad T_{l i j}=\frac{\partial}{\partial x_{l}} \psi_{i j}
$$

The friction force is due to the second term of the right-hand side of Equation (3), and for dilute media, the first term can be neglected. However, for the more general situation to be considered below, this will no longer be the case, since correlations will be induced.

For the time-dependent part of Equation (3), we may write $-A F(t) \rightarrow-A_{l} F_{l}(t)$, where $A_{l}=B_{l}$ and $F_{l}(t)=v_{l} t$. According to Kubo [20,21,25], the perturbing term leads to a response in the thermal average of $B_{l}$, given by:

$$
\Delta\left\langle B_{l}(t)\right\rangle=\int_{-\infty}^{\infty} \phi_{B A l q}\left(t-t^{\prime}\right) F_{q}\left(t^{\prime}\right) d t^{\prime}
$$

where the response function is $(t>0)$ :

$$
\phi_{B A l q}(t)=\frac{1}{i \hbar} \operatorname{Tr}\left\{\rho\left[A_{q}, B_{l}(t)\right]\right\}
$$

Here, $\rho$ is the density matrix and $B_{l}(t)$ is the Heisenberg operator, $B_{l}(t)=e^{i t H / \hbar} B_{l} e^{-i t H / \hbar}$, where $B_{l}$, like $A_{q}$, are time-independent operators. With Equations (3) and (4), expression (6) can be rewritten as:

$$
\phi_{B A l q}(t)=G_{\text {lqijnm }} \phi_{i j n m}(t)
$$

where:

$$
\begin{gathered}
G_{l q i j n m}=T_{l i j} T_{q n m} \\
\phi_{i j n m}(t)=\operatorname{Tr}\left\{\rho C_{i j n m}(t)\right\} \\
C_{i j n m}(t)=\frac{1}{i \hbar}\left[s_{1 i} s_{2 j}, s_{1 n}(t) s_{2 m}(t)\right]
\end{gathered}
$$

(the $i$ in the denominator is the imaginary unit).

Here, as in [18], it is convenient to use imaginary time, $\lambda$, and consider the correlation function:

$$
g_{i j n m}(\lambda)=\operatorname{Tr}\left[\rho s_{1 n}(t) s_{2 m}(t) s_{1 i} s_{2 j}\right]
$$

with $\lambda=i t / \hbar$. 
The key problem when dealing with media with general permittivity will be the evaluation of this function to obtain the friction force. For dilute media, however, the two oscillators (assumed isotropic) are independent, so we have:

$$
\begin{gathered}
g_{i j n m}(\lambda)=g_{1 i n}(\lambda) g_{2 j m}(\lambda) \\
g_{a p q}(\lambda)=\left\langle s_{a q}(t) s_{a p}\right\rangle=g_{a}(\lambda) \delta_{q p} \quad(a=1,2)
\end{gathered}
$$

where the angular brackets denote thermal averages, $(\langle.\rangle=.\operatorname{Tr}[\rho .]$.$) .$

The $\phi$ is related to the $g$ via:

$$
\phi_{i j n m}(t)=\frac{1}{i \hbar}\left[g_{i j n m}(\beta+\lambda)-g_{i j n m}(\lambda)\right]
$$

and:

$$
\tilde{\phi}(\omega)=\tilde{g}(K)
$$

where the Fourier transforms are:

$$
\begin{aligned}
& \tilde{\phi}(\omega)=\int_{0}^{\infty} \phi(t) e^{-i \omega t} d t \\
& \tilde{g}(K)=\int_{0}^{\beta} g(\lambda) e^{i K \lambda} d \lambda
\end{aligned}
$$

with $K$, the imaginary frequency:

$$
K=i \hbar \omega
$$

Here, $\beta=1 / k_{B} T$, where $T$ is the temperature and $k_{B}$, Boltzmann's constant.

With Equations (12) and (13), we have:

$$
\begin{gathered}
g_{i j n m}(\lambda)=g(\lambda) \delta_{i n} \delta_{j m} \\
g(\lambda)=g_{1}(\lambda) g_{2}(\lambda)
\end{gathered}
$$

by which the $\tilde{g}(K)$ can be written as a convolution:

$$
\tilde{g}(K)=\frac{1}{\beta} \sum_{K_{0}} \tilde{g}_{1}\left(K_{0}\right) \tilde{g}_{2}\left(K-K_{0}\right)
$$

$K_{0}=2 \pi n / \beta$ ( $n$ is integer) being the Matsubara frequencies. The $\tilde{g}_{a}(K)$ can be identified with the frequency-dependent polarizability, $\alpha_{a K}$ of oscillator $a(=1,2)$, which, for a simple harmonic oscillator, is:

$$
\tilde{g}_{a}(K)=\alpha_{a K}=\frac{\alpha_{a}\left(\hbar \omega_{a}\right)^{2}}{K^{2}+\left(\hbar \omega_{a}\right)^{2}}
$$

where $\alpha_{a}$ is the zero-frequency polarizability.

With Equations (15) and (19), the Fourier transform of expression (9) will be:

$$
\tilde{\phi}_{i j n m}(\omega)=\tilde{\phi}(\omega) \delta_{i n} \delta_{j m}
$$

where we recall that $\tilde{\phi}(\omega)=\tilde{g}(K)$. 
Further, following [18], the friction force is given by:

$$
\begin{gathered}
F_{f l}=-G_{l q} v_{q} \int_{0}^{\infty} \phi(u) u d u=-\left.i G_{l q} v_{q} \frac{\partial \tilde{\phi}(\omega)}{\partial \omega}\right|_{\omega=0} \\
=-G_{l q} v_{q} H \frac{\pi \beta}{2} \delta\left(\omega_{1}-\omega_{2}\right)
\end{gathered}
$$

where:

$$
\begin{gathered}
G_{l q}=G_{l q i i j j}=T_{l i j} T_{q i j} \\
H=\left(\frac{m}{2 \sinh \left(\frac{1}{2} \beta m\right)}\right)^{2} \alpha_{1} \alpha_{2}
\end{gathered}
$$

with $m=\hbar \omega$, where $\omega_{1}-\omega_{2}=\omega$.

The treatment above can be extended to a more general polarizability:

$$
\alpha_{a}(K)=\tilde{g}_{a}(K)=f\left(K^{2}\right)
$$

where it can be shown that the function, $f\left(K^{2}\right)$, satisfies the relation [26]:

$$
f\left(K^{2}\right)=\int \frac{\alpha_{I a}\left(m^{2}\right) m^{2}}{K^{2}+m^{2}} d\left(m^{2}\right)
$$

with:

$$
\alpha_{I a}\left(m^{2}\right) m^{2}=-\frac{1}{\pi} \Im\left[f\left(-m^{2}+i \gamma\right)\right], \quad\left(m=\hbar \omega=-i K, \gamma \rightarrow 0^{+}\right)
$$

With this, one finds:

$$
\begin{gathered}
F_{f l}=-G_{l q} v_{q} H_{0} \\
H_{0}=\frac{\pi \beta \hbar^{2}}{2} \int \frac{m^{4} \alpha_{I 1}\left(m^{2}\right) \alpha_{I 2}\left(m^{2}\right)}{\sinh ^{2}\left(\frac{1}{2} \beta m\right)} d \omega
\end{gathered}
$$

This is obtained by replacing $\alpha_{a}$ with $\alpha_{I a}\left(m_{a}^{2}\right) d\left(m_{a}^{2}\right)(a=1,2)$ in expression (26), which is then inserted in Equation (24) and, then, integrated with the $\delta$-function included.

Finally, by integrating $G_{l q}$ over space, one obtains for dilute media the friction force, $F_{h}$, between a particle and a half-plane, and the friction force, $F$ (per unit area), between two half-planes that move parallel to each other:

$$
F_{h}=-G_{h} v H_{0}, \quad \text { and } \quad F=-G v H_{0}
$$

Here, $v$ is the relative velocity in the $x$ direction, and one finds [18]:

$$
\begin{gathered}
G_{h}=\rho_{1} \int_{z>z_{0}} G_{11} d x d y d z=\frac{3 \pi \rho_{1}}{2 z_{0}^{5}} \\
G=\rho_{2} \int_{d}^{\infty} G_{h} d z=\frac{3 \pi}{8 d^{4}} \rho_{1} \rho_{2}
\end{gathered}
$$

Here, $\rho_{1}$ and $\rho_{2}$ are the particle densities in the half-planes, $z_{0}$ is the separation between the particle and one half-plane and $d$ is the separation between the half-planes. 


\section{Use of Fourier Methods}

For higher densities of polarizable particles, the above results will be modified, due to induced dipolar correlations within planes and between planes. This affects the evaluations of $G_{h}$ and $G$, which become more complex and demanding. Further, expression (31) for $H_{0}$ will be modified, where we will find that the imaginary parts of the polarizabilities will be replaced by functions based upon the frequencydependent permittivity only. Thus, the friction will depend solely on this macroscopic property and be independent of the explicit relation between the permittivity and the polarizability. On physical grounds, we find this reasonable.

To facilitate the analysis, we find it convenient to evaluate the integral (33) by use of a Fourier transform in the $x$ and $y$ directions. Then, the quantities in Equations (2)-(4) should be transformed. The three-dimensional Fourier transform of the Coulomb potential, $\psi=1 / r$, is:

$$
\tilde{\psi}(k)=\frac{4 \pi}{k^{2}}, \quad k^{2}=k_{i} k_{i}
$$

where $k_{i}(i=1,2,3$ or $x, y, z)$ is the Fourier variable. This can be transformed back with respect to $z$ to obtain:

$$
\hat{\psi}\left(z, k_{\perp}\right)=\frac{2 \pi e^{-i k_{z} z}}{k_{\perp}}=\frac{2 \pi e^{-q|z|}}{k_{\perp}}
$$

where $q=k_{\perp}, k_{\perp}^{2}=k_{x}^{2}+k_{y}^{2}$, and $i k_{z}= \pm q$ for $z>0$ or $z<0$. The variable, $k_{z}$, may seem unnecessary here, but it is kept for convenience, in order to obtain simple and compact expressions for the transforms of derivatives. Otherwise, one would need separate expressions for the transforms in the $x$ and $y$ directions and in the $z$ direction.

Thus, with Equations (2) and (4), $\left(\partial / \partial x_{j} \rightarrow-i k_{j}\right)$ :

$$
\begin{gathered}
\hat{\psi}_{i j}=\hat{\psi}_{i j}\left(z, \mathbf{k}_{\perp}\right)=-k_{i} k_{j} \hat{\psi} \\
\hat{T}_{l i j}=\hat{T}_{l i j}\left(z, \mathbf{k}_{\perp}\right)=-i k_{l} k_{i} k_{j} \hat{\psi}
\end{gathered}
$$

with this, the $x y$-integration of Equation (33) becomes:

$$
G_{\perp}=\int G_{11} d x d y=\frac{1}{(2 \pi)^{2}} \int \hat{G}_{11} d \mathbf{k}_{\perp}
$$

where expression (25) for $G_{l q}$ transforms into:

$$
\hat{G}_{l q}=\hat{T}_{l i j}\left(z, \mathbf{k}_{\perp}\right) \hat{T}_{q i j}\left(z,-\mathbf{k}_{\perp}\right)=k_{l} k_{q} k_{i} k_{i} k_{j} k_{j} \hat{\psi}^{2}
$$

Here, some care must be taken in the summations as $i k_{z}$ follows the sign of $z$ :

$$
-i k_{j} \times i k_{j}=k_{x}^{2}+k_{y}^{2}+( \pm q)^{2}=k_{\perp}^{2}+q^{2}=2 q^{2}
$$

With this, we find:

$$
\hat{G}_{11}=k_{x}^{2}\left(2 q^{2}\right)^{2} \hat{\psi}^{2}
$$

Symmetry with respect to $x$ and $y$ means that $k_{x}^{2}$ can be replaced by $\frac{1}{2}\left(k_{x}^{2}+k_{y}^{2}\right)=\frac{1}{2} k_{\perp}^{2}=\frac{1}{2} q^{2}$ in the integral (39), so we get, $(z>0)$ :

$$
G_{\perp}=\frac{1}{(2 \pi)^{2}} \int_{0}^{\infty} 2 q^{6}\left(\frac{2 \pi e^{-q z}}{q}\right)^{2} 2 \pi q d q=4 \pi \frac{5 !}{2^{6} z^{6}}=\frac{15 \pi}{2 z^{6}}
$$


By further insertion into Equations (33) and (34), the results of those integrals are recovered.

\section{General Density}

\subsection{Half-Planes Considered As Composite Particles}

For higher densities, separate oscillators both within each plane and between planes will be correlated. This will add to the complexity of the problem. However, the structure of the problem can be simplified by regarding the two half-planes as a generalized version of a pair of particles. Some details of this approach are given a closer treatment in Appendix A.

Expression (11) is a thermal average of four oscillating dipole moments. They have Gaussian distributions, since they represent coupled harmonic oscillators. This means that averages can be divided into averages of pairs of dipole moments. Thus, we have

$$
\begin{gathered}
g_{i j n m}(\lambda)=\left\langle s_{1 n}(t) s_{2 m}(t) s_{1 i} s_{2 j}\right\rangle=\left\langle s_{1 n}(t) s_{2 m}(t)\right\rangle\left\langle s_{1 i} s_{2 j}\right\rangle \\
+\left\langle s_{1 n}(t) s_{1 i}\right\rangle\left\langle s_{2 m}(t) s_{2 j}\right\rangle+\left\langle s_{1 n}(t) s_{2 j}\right\rangle\left\langle s_{2 m}(t) s_{1 i}\right\rangle
\end{gathered}
$$

Now, the first term on the right-hand side is the equal-time average of the operators, $A$ and $B, \sim s_{1 i} s_{2 j}$, and should be subtracted from Equation (9) to obtain the proper response function. Thus, we need:

$$
\Delta g_{i j n m}(\lambda)=\left\langle s_{1 n}(t) s_{1 i}\right\rangle\left\langle s_{2 m}(t) s_{2 j}\right\rangle+\left\langle s_{1 n}(t) s_{2 j}\right\rangle\left\langle s_{2 m}(t) s_{1 i}\right\rangle
$$

Here, the first average represents correlations within the same half-plane, while the second is the same for different planes.

To better see the structure or formal contributions to these correlations, one may consider the free energy of a pair of one-dimensional oscillators. The harmonic fluctuations result in a distribution function for the dipole moment of each molecule, assuming a static polarizability, $\alpha$,

$$
\rho(s)=\exp \left(-\frac{\beta s^{2}}{2 \alpha}\right)
$$

If the configuration becomes perturbed by an interaction energy, $\phi s_{1} s_{2}$ (not necessarily equal to the Coulomb potential, called $\psi$ above), the partition function is:

$$
Z=\int d s_{1} d s_{2} \rho\left(s_{1}\right) \rho\left(s_{2}\right) e^{-\beta \phi s_{1} s_{2}}
$$

Thus, the change in the free energy, $F$, due to the mutual interaction, $\phi s_{1} s_{2}$, becomes (In [21], this expression was used as a basis with $\alpha_{2}=\alpha_{1}$ ):

$$
\ln Z=-\beta F=-\frac{1}{2} \ln \left(1-\alpha_{1} \alpha_{2} \phi^{2}\right)
$$

where $\alpha_{1}$ and $\alpha_{2}$ are the polarizabilities of the two oscillators. The correlation functions, $\left\langle s_{a}^{2}\right\rangle(a=1,2)$ and $\left\langle s_{1} s_{2}\right\rangle$ are:

$$
\beta\left\langle s_{a}^{2}\right\rangle=\frac{\alpha_{a}}{1-\alpha_{1} \alpha_{2} \phi^{2}}
$$




$$
\beta\left\langle s_{1} s_{2}\right\rangle=\frac{Z^{\prime}}{Z}=I^{\prime}=\frac{\alpha_{1} \alpha_{2} \phi}{1-\alpha_{1} \alpha_{2} \phi^{2}}
$$

where the prime means differentiation with respect to $\phi$. Further:

$$
\begin{gathered}
\beta^{2}\left(\left\langle s_{1} s_{2} s_{1} s_{2}\right\rangle-\left\langle s_{1} s_{2}\right\rangle\left\langle s_{1} s_{2}\right\rangle\right)=\beta^{2}\left(\left\langle s_{1}^{2}\right\rangle\left\langle s_{2}^{2}\right\rangle+\left\langle s_{1} s_{2}\right\rangle\left\langle s_{1} s_{2}\right\rangle\right) \\
=\frac{Z^{\prime \prime}}{Z}-\left(\frac{Z^{\prime}}{Z}\right)^{2}=I^{\prime \prime}=\frac{\alpha_{1} \alpha_{2}}{1-\alpha_{1} \alpha_{2} \phi^{2}}+\frac{2\left(\alpha_{1} \alpha_{2} \phi\right)^{2}}{\left(1-\alpha_{1} \alpha_{2} \phi^{2}\right)^{2}} \\
=\frac{\alpha_{1}}{1-\alpha_{1} \alpha_{2} \phi^{2}} \frac{\alpha_{2}}{1-\alpha_{1} \alpha_{2} \phi^{2}}+\frac{\alpha_{1} \alpha_{2} \phi}{1-\alpha_{1} \alpha_{2} \phi^{2}} \frac{\alpha_{1} \alpha_{2} \phi}{1-\alpha_{1} \alpha_{2} \phi^{2}}
\end{gathered}
$$

When extended to half-planes, one may interpret this expression in the following way: In the first term, on the right-hand side, $\alpha_{1}$ and $\alpha_{2}$ are the correlations within each half-plane for $\phi=0$. When the planes interact, the denominator represents induced correlations, due to the presence of the second plane. Likewise, the second term in Equation (47) represents correlations between the planes.

From a fundamental point of view, the following feature should, however, be noted here. The interaction, $\phi$, in Equation (47) will shift the eigenfrequencies of two oscillators further apart. Each term in Equation (47) will contain both these new frequencies when expanded. For each frequency, we can, in principle, repeat the evaluation that led to Equation (24). However, now, $\omega_{1} \neq \omega_{2}$, and the delta function will not contribute. For the situation with low constant relative velocity, there will accordingly be no friction. This is also reasonable, since excitation of the quantized system requires disturbances with frequencies that match the energy difference, $\hbar\left(\omega_{1}-\omega_{2}\right)$, while low constant velocity represents the limit of zero frequencies. However, with continuous frequency bands in each oscillator, there may again be equal frequencies, $\omega_{1}=\omega_{2}$, for the perturbed system.

It is possible to interpret expressions (45)-(47) in terms of graphs. When expanded, each term in Equation (45) represents a closed ring with $\alpha_{1}$ and $\alpha_{2}$ vertices that are connected by $\phi$-bonds. In expression (46) for $\left\langle s_{1} s_{2}\right\rangle$, one $\phi$-bond is taken out from the ring by which one gets a chain of bonds ending on different vertices. In Equation (47), another $\phi$-bond is taken out from the ring, which is then split into two chains for which there will be two different situations consistent with the expression on the right-hand side of this equation: in one case, each chain has both its endpoints on the same particle, while in the other case, the chains have their endpoints on different particles.

For our problem, we also need correlations in imaginary time, $\lambda=i t / \hbar$, as in Equations (42) and (43). This generalizes Equation (47) into $\left(s_{a}=s_{a}(0)\right)$ :

$$
\begin{aligned}
h(\lambda) & =\left\langle s_{1}(t) s_{2}(t) s_{1} s_{2}\right\rangle-\left\langle s_{1}(t) s_{2}(t)\right\rangle\left\langle s_{1} s_{2}\right\rangle \\
& =h_{11}(\lambda) h_{22}(\lambda)+h_{12}(\lambda) h_{21}(\lambda)
\end{aligned}
$$

where $\left(s_{a b}=s_{b a}\right)$ :

$$
h_{a b}(\lambda)=\left\langle s_{a}(t) s_{b}\right\rangle
$$

Its Fourier transform in imaginary time is, similar to Equation (21):

$$
\tilde{h}(K)=\frac{1}{\beta} \sum_{K_{0}}\left[\tilde{h}_{11}\left(K_{0}\right) \tilde{h}_{22}\left(K-K_{0}\right)+\tilde{h}_{12}\left(K_{0}\right) \tilde{h}_{21}\left(K-K_{0}\right)\right]
$$


In view of the graph interpretation, the structure of Equation (50) will be similar to the one of Equation (47), so:

$$
\begin{gathered}
\tilde{h}_{a a}(K)=\frac{\alpha_{a K}}{1-\alpha_{1 K} \alpha_{2 K} \phi^{2}}, \quad(a=1,2) \\
\tilde{h}_{12}(K)=\frac{\alpha_{1 K} \alpha_{2 K} \phi}{1-\alpha_{1 K} \alpha_{2 K} \phi^{2}}
\end{gathered}
$$

With static interactions, the $\phi$ will not vary with $K$.

With three-dimensional polarizations, the formalism will be modified, but will still be manageable. Some of the points will be verified more explicitly in Appendix A.

As established in [27], the correlation functions for polarizations will be solutions of Maxwell's equations. For dielectric half-planes with permittivities, $\varepsilon_{1}=\varepsilon_{1}(K)$ and $\varepsilon_{2}=\varepsilon_{2}(K)$, one finds (cf., Appendix A):

$$
\alpha_{1 K} \alpha_{2 K} \phi^{2} \rightarrow A_{1 K} A_{2 K} e^{-2 q d}, \quad A_{a K}=\frac{\varepsilon_{a}-1}{\varepsilon_{a}+1}
$$

where $d$ is the separation between the planes.

In Equation (51), $\alpha_{a K}$ alone represents correlations within each plane. For dilute media, it corresponds to the polarizability, whose relation to the permittivity is:

$$
\varepsilon_{a}-1=4 \pi \rho_{a} \alpha_{a K}
$$

consistent with Equation (52). As verified in Appendix A, it follows that for a general permittivity, the $\alpha_{a K}$ in Equation (51) will generalize to:

$$
4 \pi \rho_{a} \alpha_{a K} \rightarrow \frac{2\left(\varepsilon_{a}-1\right)}{\varepsilon_{a}+1}=2 A_{a K}
$$

and by that:

$$
\phi \rightarrow 2 \pi\left(\rho_{1} \rho_{2}\right)^{1 / 2} e^{-q d}
$$

Consistent with Equation (52), Equation (51) is modified to:

$$
\begin{gathered}
2 \pi \rho_{a} \tilde{h}_{a a}(K)=\frac{A_{a K}}{1-A_{1 K} A_{2 K} e^{-2 q d}} \\
2 \pi\left(\rho_{1} \rho_{2}\right)^{1 / 2} \tilde{h}_{12}(K)=\frac{A_{1 K} A_{2 K} e^{-q d}}{1-A_{1 K} A_{2 K} e^{-2 q d}}
\end{gathered}
$$

The function, $\tilde{h}(K)$, given by Equation (50) will, in the general case, replace the $\tilde{g}(K)$ given by Equation (21). Again, one may decompose into contributions from harmonic oscillators, as done in Equations (27)-(31). Then, Equation (31) transforms into:

$$
\begin{aligned}
& H_{0} \rightarrow H_{0}(u)=\frac{\pi \beta \hbar^{2}}{2} \int \frac{m^{4}}{\sinh ^{2}\left(\frac{1}{2} \beta m\right)} \\
& \times\left[\alpha_{I 11}\left(m^{2}, u\right) \alpha_{I 22}\left(m^{2}, u\right)+\alpha_{I 12}\left(m^{2}, u\right) \alpha_{I 12}\left(m^{2}, u\right)\right] d \omega
\end{aligned}
$$

where:

$$
\alpha_{I a b}\left(m^{2}, u\right) m^{2}=-\frac{1}{\pi} \Im\left(f\left(-m^{2}+i \gamma\right)\right]
$$




$$
\begin{gathered}
f\left(K^{2}\right)=\tilde{h}_{a b}(K), \quad(a, b=1,2) \\
u=q d
\end{gathered}
$$

For one particle outside a half-plane, one has $\rho_{1} \rightarrow 0$ and, thus, $A_{1 K} \rightarrow 0$ (when $\rho_{2}$ is the density in the half-plane). With this, the numerators in the expressions are replaced by one, and $\tilde{h}_{12}(K)$ vanishes, so in this case, the $H_{0}(u)$ will no longer vary with $x$. The same situation occurs when medium 1 is dilute. Due to this, the expressions (32) for the friction force will be the same, except that in expression (31) for $H_{0}$, the role of $\alpha_{2 K}$ is replaced by $A_{2 K}$, as given by Equation (54). Thus:

$$
\alpha_{2 K} \rightarrow \frac{\varepsilon_{2}-1}{2 \pi \rho_{2}\left(\varepsilon_{2}+1\right)}
$$

while $\alpha_{1 K}$ is kept.

For larger $\alpha_{1 K}$, the $u$-dependence will be present. This will modify the integral (41) as $H_{0}(u)$ has to be included. However, the $z$ - and $z_{0}$-integration over the half-planes that led to the results, (33) and (34), can be evaluated first. With $G_{\perp}$ given by integral (41), we then find $(u=q d)$ :

$$
\int_{d}^{\infty}\left(\int_{z>z_{0}} G_{\perp} d z\right) d z_{0}=\frac{\pi}{d^{4}} \int_{0}^{\infty} u^{3} e^{-2 u} d u=\frac{3 \pi}{8 d^{4}}
$$

With this, the $H_{0}$ can be included along with the integrand of Equation (59), and the friction force, $F$, between two half-planes can again be written in the form of Equation (32) as:

$$
F=-G v H_{0}
$$

where $G$, again, is given by Equation (34) as $G=3 \pi \rho_{1} \rho_{2} /\left(8 d^{4}\right)$, but where now:

$$
H_{0}=\frac{8}{3} \int_{0}^{\infty} u^{3} H_{0}(u) e^{-2 u} d u
$$

with $H_{0}(u)$, given by Equation (56).

\subsection{Further Comments on the Complexities Coming from Internal Interactions in the Planes}

Central equations in our context are Equations (47) and (55). However, their direct use will present certain problems, as the interaction, $\phi$, in Equation (47) will shift the frequencies of the two oscillators to new eigenfrequencies further apart from each other. Thus, when repeating the evaluations that led to Equation (24), there will no longer be contribution to the friction force at all, since the $\delta$-function will vanish. (Each of the two factors in the two terms of Equation (47) will then also contain equal frequencies, but they will not contribute to the limiting procedure that led to Equation (24) [18].) Therefore, for the situation in which the constant relative velocity is low, there will be no friction. (Continuous frequency bands in each oscillator may change this, but we will not investigate this further here.) This absence of friction may somehow be reasonable for the following physical reason: Excitations of the quantized system require disturbances with frequencies that match the energy difference, $\hbar\left(\omega_{1}-\omega_{2}\right)$, while low constant velocity represents the limit of zero frequency. Moreover, the expansion (3) requires the displacement to be small and comparable to molecular diameters, a situation that may be of minor interest. 
Due to these problems, we find it more appropriate and realistic to consider the whole mutual interaction, $\phi$, as belonging to the perturbing force. An obvious justification for this is that the mutual interaction, $\phi=0$, when the two particles are far apart for $t \rightarrow \pm \infty$. Then, we are back to our previous situation, where the left-hand side of Equation (47) reduces to $\alpha_{1} \alpha_{2}$, i.e., the nominator in the first term on the right-hand side. In the presence of half-spaces, the situation is less trivial, however, due to interactions within each half-plane. Then, the $A_{a K}$ given by Equation (52) will replace $\alpha_{a K}(a=1,2)$. The precise replacement is given by Equation (58).

Next, it is noteworthy that the expression (58) can be given a direct physical interpretation. This is most obvious for metals, where the dielectric constant is given by the plasma relation $\left(\varepsilon=\varepsilon_{a}\right)$ :

$$
\varepsilon=1-\left(\frac{\omega_{p}}{\omega}\right)^{2}
$$

the $\omega_{p}$ being the plasma frequency. Then, with Equation (58):

$$
2 \pi \rho \alpha_{K} \rightarrow \frac{\varepsilon-1}{\varepsilon+1}=\frac{\omega_{p}^{2}}{\omega_{p}^{2}-2 \omega^{2}}
$$

which corresponds to the polarizability of a harmonic oscillator with eigenfrequency:

$$
\omega=\frac{\omega_{p}}{\sqrt{2}}
$$

Thus, each half-plane represents a set of harmonic oscillators for each wavevector, $\mathbf{k}_{\perp}$, in the $x y$-plane. In the idealized situation considered here, they all have the same eigenfrequency.

The above mentioned result Equation (64) can be given a simple physical interpretation, as it represents the frequency of surface plasma waves [23]. Thus, each half-plane can be regarded as an assembly of independent harmonic oscillators. There is an oscillator for each wavevector, $\mathbf{k}_{\perp}$, and they all oscillate with the frequency of surface plasma waves. With these waves, there is a net charge that oscillates on the surface, while the inside of the half-plane is neutral. The corresponding electric potential (apart from its time dependence) is given by Equation (A.1), where the coefficients, $B$ and $C$ (for $d \rightarrow \infty$ ), diverge for the frequency of surface plasma waves.

\section{Numerical Examples and Comparison with Earlier Works}

Casimir friction is a complicated topic; it is approached from different points of view by various researchers, and the obtained results are not always so easy to compare with each other.

The basic method used by us is to employ statistical mechanical methods based upon the Kubo formula. The simplest systems to compare are clearly those of low particle density. The approach most closely related to ours for such a case appears to be that of Barton [22-24]. Even then, it turns out that the methods are not easily comparable; $c f$., the remarks of Barton himself given in [24]. In the paper [17], we showed, however, by a detailed calculation, that the results of Barton and those of ours are actually in agreement. This circumstance lends credence to our approach and indicates that we are on the right track.

The essential new development of the present paper is to extend the statistical mechanical method to the case of finite density. As far as we know, such a treatment has not been given before. Further, it is 
of obvious interest to try to compare our results with those obtained by others; results that have been derived via different methods. Results obtained by others are usually based upon methods of quantum electrodynamics of continuous media and contain macroscopic concepts, such as electrical conductivity.

Now, let us consider metal plates for which the permittivity, $\varepsilon$, is given by the plasma relation (62) as an idealized case. As a physical quantity, the $\varepsilon$ with dispersion will have to include also an imaginary part for real $\omega$. The simplest generalized version of the expression (62) is the one of the Drude model:

$$
\varepsilon=1+\frac{\omega_{p}^{2}}{\zeta(\zeta+\nu)}
$$

where $\zeta=i \omega$ and where $\nu$ represents damping of plasma oscillations, due to finite conductivity of the medium. (Note that a different convention implying $\zeta=-i \omega$ is frequently used, the sign being dictated by the Fourier transform used. We here assume $\tilde{f}(\omega)=\int f(t) e^{-i \omega t} d t$, such that singularities will only be present for $\Im(\omega)>0$, with $f(t)=0$ for $t<0$, due to causality.)

In terms of the variable $K=i \hbar \omega=\hbar \zeta$, Equation (65) can be written as:

$$
\varepsilon=1+\frac{2 q^{2}}{K^{2}+\sigma|K|}
$$

where:

$$
q^{2}=\frac{\left(\hbar \omega_{p}\right)^{2}}{2} \text { and } \sigma=\hbar \nu
$$

have been introduced. The $\varepsilon$ as a function of either $\omega$ or $K$ is the same in the common region, where $\varepsilon$ has no singularities, i.e., for $\Im(\omega)<0$ or $\Re(K)>0$ [21]. Further, $\varepsilon(K)$ is symmetric in $K$, and $|K|$ is to be interpreted as $|K|=\lim \left(K^{2}+\gamma^{2}\right)^{1 / 2}, \gamma \rightarrow 0$. The singularities representing the singularities of $\varepsilon(K)$ will be along the imaginary $K$-axis or for $K^{2}$ negative.

For finite density, expression (58) is to be formed. Therefore, with Equation (66) for $\varepsilon$, expression (63) is modified into:

$$
2 \pi \rho \alpha_{K} \rightarrow \frac{\varepsilon-1}{\varepsilon+1}=\frac{q^{2}}{K^{2}+q^{2}+\sigma|K|}
$$

The evaluation of the friction now follows from Equations (27)-(34). By inserting expression (67) in Equation (29), the frequency spectrum is obtained. With $K=i m$, we have $(\gamma \rightarrow 0+)$ :

$$
2 \pi \rho \alpha_{K} \rightarrow \frac{q^{2}\left(-m^{2}+q^{2}-i \sigma m\right)}{\left(-m^{2}+q^{2}\right)^{2}+(\sigma m)^{2}}
$$

which gives the frequency spectrum:

$$
2 \pi \rho \alpha_{I}\left(m^{2}\right) m^{2}=-\frac{1}{\pi} \Im\left(f\left(-m^{2}\right)\right)=\frac{q^{2}}{\pi} \frac{\sigma m}{\left(-m^{2}+q^{2}\right)^{2}+(\sigma m)^{2}}
$$

For small $\sigma$, we can simplify this, as the expression will be sharply peaked around $m=q$. Thus, one might assume to get the main contribution from around this value. However, unless $\beta m$ is small, the $\sinh (\cdot)$ term in Equation (31) will be very large, by which values around $m=q$ can be fully neglected; and for small $m$, Equation (69) can be replaced by:

$$
m^{2} \alpha_{I}\left(m^{2}\right)=D m, \quad D=\frac{\sigma}{2 \pi^{2} \rho q^{2}}
$$


This is to be inserted in Equation (31) to obtain $\left(\alpha_{I 1}=\alpha_{I 2}=\alpha_{I}: d m=\hbar d \omega\right)$

$$
\begin{aligned}
H_{0} & =\frac{\pi \beta \hbar}{2} D^{2} \int_{0}^{\infty} \frac{m^{2} d m}{\sinh ^{2} \frac{1}{2} \beta m}=\frac{2 \pi \hbar}{\beta^{2}} D^{2} I \\
I & =\int_{0}^{\infty} \frac{x^{2} e^{-x} d x}{\left(1-e^{-x}\right)^{2}}=\sum_{n=1}^{\infty} \int_{0}^{\infty} x^{2} n e^{-n x} d x=2 ! \sum_{n=1}^{\infty} \frac{1}{n^{2}}=\frac{\pi^{2}}{3}
\end{aligned}
$$

where the substitution, $x=\beta m$, has been made.

Finally, with Equations (32) and (34), we find for the friction force per unit area $\left(\rho_{1}=\rho_{2}=\rho\right.$, and $k_{B}$ is Boltzmann's constant):

$$
\begin{aligned}
F & =-G v H_{0}=-\frac{3 \pi}{8 d^{4}} \rho^{2} v H_{0}=-\frac{3 \pi}{8 d^{4}} \rho^{2} v \frac{2 \pi \hbar}{\beta^{2}} D^{2} I \\
& =-\frac{v\left(k_{B} T\right)^{2} \hbar \sigma^{2}}{16 d^{4} q^{4}}=-\frac{\hbar v}{4 d^{4}} \frac{\left(k_{B} T\right)^{2}(\hbar \nu)^{2}}{\left(\hbar \omega_{p}\right)^{4}}
\end{aligned}
$$

Let us consider a numerical example. Assume room temperature, $T=300 \mathrm{~K}$, corresponding to $k_{B} T=25.86 \mathrm{meV}$. Choose gold as the medium, for which $\hbar \omega_{p}=9.0 \mathrm{eV}$ and $\hbar \nu=35 \mathrm{meV}$. Then, choose $v=100 \mathrm{~m} / \mathrm{s}$ for the relative velocity and a small separation, $d=10 \mathrm{~nm}$, between the plates. With $\hbar=1.054 \times 10^{-34} \mathrm{Js}$, we then find for the friction force (72):

$$
F=3.29 \times 10^{-11} \mathrm{~Pa}
$$

This is a very small force. However, by changing parameters, this will change rapidly by which $F$ can become very large instead. However, first, let us compare this force with the result obtained by Pendry [2] for $T=0$, where the friction linear in velocity was found to be zero, assuming constant conductivity. Instead, a non-zero force, proportional to $v^{3}$, was found. The influence of relative velocity is to create an oscillating force between the particles; these oscillations will create excitations from the $T=0$ ground states of low frequency oscillations and thus contribute to friction. According to the derivations of [2], the friction for low and not too high velocities for a dielectric function, $\varepsilon=1+i \sigma / \omega \varepsilon_{0}$, was found to be:

$$
F_{P}=\frac{5 \hbar \varepsilon_{0}^{2} v^{3}}{2^{8} \pi^{2} \sigma^{2} d^{6}}
$$

(here and henceforth, $\sigma$ is the conductivity in SI units, not $\hbar \nu$, as above). When compared with our dielectric function Equation (65), for small $\omega$, one sees that the $\sigma / \varepsilon_{0}$ of this equation is our $\omega_{p}^{2} / \nu$. This can then be inserted in Equation (74), and one finds the ratio between the friction forces, (72) and (74), to be:

$$
\frac{F}{F_{P}}=\frac{64 \pi^{2}}{5}\left(\frac{k_{B} T}{\hbar v / d}\right)^{2}
$$

This simple expression can be given a direct physical interpretation. The $k_{B} T$ is the energy quanta that can be excited, due to thermal energies or fluctuations, while $\hbar v / d$ are the energy quanta generated, due to frequencies, $v / d$, generated by the finite velocity. Due to the physical interpretation above and arguments used in [2], there is reason to expect its result for finite velocity and zero temperature to be 
consistent with ours, obtained for small velocity and nonzero temperature. For the numerical values that gave the force Equation (73), one finds the ratio:

$$
\frac{F}{F_{P}}=1.95 \times 10^{9}
$$

Thus, thermal energies have a much greater influence than the frequencies generated from the finite velocity. In [2], a much greater friction was obtained by using other numerical values. There, $\sigma=0.1 \Omega^{-1} \mathrm{~m}^{-1}, d=10^{-10} \mathrm{~m}$ and $v=1.0 \mathrm{~m} / \mathrm{s}$ was used. With permittivity of vacuum, $\varepsilon_{0}=8.85 \times 10^{-12} \mathrm{As} / \mathrm{Vm}$, one then finds $\omega_{p}^{2} / \nu=\sigma / \varepsilon_{0}=1.12 \times 10^{10} \mathrm{~s}^{-1}$, while result (73) was based upon $\sigma / \varepsilon_{0}=\omega_{p}^{2} / \nu=3.5 \cdot 10^{18} \mathrm{~s}^{-1}$, a much greater number. The numbers of [2] will, thus, give the very large friction force:

$$
F=3.5 \cdot 10^{12} \mathrm{~Pa}
$$

which, with the ratio (75), means (as $v / d$ is unchanged) $F_{P}=1.6 \times 10^{3} \mathrm{~Pa}$. In [2], the result, $F_{P} \approx 3 \times 10^{3} \mathrm{~Pa}$, was found by substitution into its high velocity formula (the formula least sensitive to velocity). Anyway, the latter force (77) is unrealistically large. One reason is that a separation, $d=10^{-10} \mathrm{~m}$, would more or less imply direct contact between the particles of the two half-planes. Further, the force decreases very rapidly with increasing separation.

Another factor of influence here is that the effective separation between the planes will increase when the charge density of electrons becomes small. This has not been taken into account. Clearly, when neglecting dielectric effects otherwise, the force should vanish when the density of electrons and, thus, $\sigma$ vanishes, while results (72) and (75) tell the opposite. In this respect, the Casimir force between parallel plates filled with a plasma of electrons was considered by us earlier (in the classic electrostatic limit) $[28,29]$. Then, it was found that the separation, $d$, increased to an effective separation, $d+2 / \kappa$ (for small densities), where $\kappa$ is the inverse shielding length, where $\kappa^{2}=4 \pi \beta e^{2} \rho$ ( $e$ is the charge of the electron) [28].

Among other previous approaches with which it seems natural to compare our results, we shall focus on those of Volokitin and Persson. As mentioned earlier, they have written a series of papers on this topic [5-9]. In contrast to the paper of Pendry [2], they considered finite temperatures. In their review article, [7], a variety of situations were considered. One such situation is for parallel relative motion of metal plates. The friction coefficient according to their Equation (97) is then:

$$
\gamma_{\| p}^{\text {evan }} \approx 0.3 \frac{\hbar}{d^{4}}\left(\frac{k_{B} T}{4 \pi \hbar \sigma}\right)^{2}
$$

where, here the conductivity, $\sigma$, is given in Gaussian units, such that $4 \pi \sigma=\omega_{p}^{2} / \nu$. This follows from their Equation (38), which is proportional to our expression (69). The friction force, $F_{V P}=\gamma_{\| p}^{\text {evan }} v$, is, thus, directly comparable to our result (72), and we find the ratio:

$$
\frac{F_{V P}}{F} \approx 1.2
$$

Thus, we can conclude that the two expressions for the friction force are consistent, except for a small difference in numerical prefactor.

A closer look at integral (92) for $\gamma_{\| p}^{\text {evan }}$ in [7] makes it probable that this difference is due to the term, $1 /(1-u)^{2}=\sum_{n=1}^{\infty} n u^{n-1}$, in the integrand with $u=e^{-2|\gamma| d}$. This term is for small $\omega \rightarrow 0$ for which 
the reflection coefficients, $R_{i p} \rightarrow 1(i=1,2)$, i.e., $(\varepsilon-1) /(\varepsilon+1) \rightarrow 1$. (Actually, when comparing with Equation (87) of that reference and with Equation (27) of [2], the $|\gamma|$ seems to be a misprint for the integration variable, q.) Then, one has integrals of the form, $\int_{0}^{\infty} n u^{n} q^{3} d q \propto n^{-3} d^{-4}$. This gives the sum:

$$
\sum_{n=1}^{\infty} \frac{1}{n^{3}}=\zeta(3)=1.202 \cdots
$$

where $\zeta(z)$ is the $\zeta$-function. This sum may be precisely the ratio (79). Here, it can be noted that the term above is also present in our expressions (47) and (55) with $u=\alpha_{1} \alpha_{2} \phi^{2}$ and $u=A_{1 K} A_{2 K} e^{-2 q d}$, respectively, but was later disregarded by further explicit evaluations, as discussed in Section 4.2.

In view of this, observing the similarity of the expressions, the results of [7] seem consistent with our results, as they agree numerically, except from some uncertainty in the prefactor.

\section{Conclusion}

A main idea of our paper has been to regard the two half-planes, sliding relatively to each other, as a generalized version of a pair of particles. With that the statistical mechanical Kubo formalism, used earlier for friction problems for a pair of particles as well as for dilute planes, can be used also in the finite density case. A central relationship in this context is Equation (52), which shows how atomic polarizabilities $\alpha_{a K}(a=1,2)$ become effectively replaced by the quantities called $A_{a K}=\left(\varepsilon_{a}-1\right) /\left(\varepsilon_{a}+1\right)$. These last quantities are, in fact, the reflection coefficients for the TM mode for small gap widths. These important relationships establish contact with results obtained by other researchers, such as Pendry [2] and others, following quite different ways of approach. Our expression for the Casimir friction force is mathematically well-defined and finite at finite temperature.

\section{Acknowledgement}

We thank Pitaevskii, L.P. for correspondence.

\section{Conflict of Interest}

The authors declare no conflict of interest.

\section{Appendix}

\section{A. Background for the Expressions for the Correlation Functions}

Here, we will justify expressions (55) for the correlation functions and the arguments that led to them. As discussed in [27], the correlation functions can be identified as solutions of Maxwell's equations. In [27] the solution was derived for two half-planes with equal permittivities. Here, we will consider two half-planes with permittivities, $\varepsilon_{1}$ and $\varepsilon_{2}$, separated by a distance, $d$. For the electrostatic case, which we will assume, the Coulomb interaction for a point charge as given in the form (36) will be the basis. 
Let the half-planes be parallel to the $x y$-plane with surfaces at $z=0$ and $z=d(>0)$. Their permittivities are $\varepsilon_{1}$ for $z<0$ and $\varepsilon_{2}$ for $z>d$. With a unit charge located at $z=z_{0}<0$, the resulting potential can be written as $\left(q=k_{z}\right)$ :

$$
\hat{\psi}\left(z, k_{\perp}\right)=\frac{2 \pi}{k_{\perp}} e^{q z_{0}} \begin{cases}\frac{1}{\varepsilon_{1}} e^{-2 q z_{0}} e^{q z}+B e^{q z}, & z<z_{0} \\ \frac{1}{\varepsilon_{1}} e^{-q z}+B e^{q z}, & z_{0}<z<0 \\ C e^{-q z}+C_{1} e^{q z}, & 0<z<d \\ D e^{-q z}, & d<z\end{cases}
$$

At the boundaries, the $\hat{\psi}$ and its derivatives, $\varepsilon_{a} \partial \hat{\psi} / \partial z(a=1,2)$, should be continuous. This yields the equations:

$$
\begin{gathered}
\frac{1}{\varepsilon_{1}}+B=C+C_{1} \\
\varepsilon_{1}\left(\frac{1}{\varepsilon_{1}}-B\right)=C-C_{1} \\
C e^{-q d}+C_{1} e^{q d}=D e^{-q d} \\
C e^{-q d}-C_{1} e^{q d}=\varepsilon_{2} D e^{-q d}
\end{gathered}
$$

These equations may first be solved for $C$ and $C_{1}$ in terms of $D$. Then, solving for $D$ and $B$, one finds:

$$
\begin{gathered}
D=\frac{4}{\left(\varepsilon_{1}+1\right)\left(\varepsilon_{2}+1\right)\left(1-A_{1 K} A_{2 K} e^{-2 q d}\right)} \\
B=\frac{1}{\varepsilon_{1}} A_{1 K}-\frac{\varepsilon_{2}-1}{\varepsilon_{1}+1} D e^{-2 q d}
\end{gathered}
$$

with $(a=1,2)$

$$
A_{a K}=\left(\varepsilon_{a}-1\right) /\left(\varepsilon_{a}+1\right)
$$

One notes that the denominator of expression (55) is the one of $D$. Further, Equation (A.4) verifies expression (54). However, to fully verify the expressions given by Equation (55) and, thus (56), the $\hat{\psi}$ of Equation (36) should be replaced with the $\hat{\psi}$ of Equation (A.1). To do this in detail, the general form of the pair correlation function for a uniform dielectric fluid as found by Høye and Stell can be used [30]. As pointed to in Equation (5.5) in [30] ( $c f$., also the more detailed considerations in [27]), this includes a prefactor, $((\varepsilon-1) /(3 y))^{2}$, with $3 y=4 \pi \rho \alpha_{K}$. With endpoints in separate half-planes, this should generalize to $((\varepsilon-1) /(3 y))^{2} \rightarrow\left(\left(\varepsilon_{1}-1\right) /\left(3 y_{1}\right)\right)\left(\left(\varepsilon_{2}-1\right) /\left(3 y_{2}\right)\right)$, where $\alpha_{K} \rightarrow \alpha_{a K}(a=1,2)$. This is then integrated with the $T_{l i j}$ of Equation (37). One will find that such an evaluation is rather nontrivial. Thus, we will not try to perform it here. Instead, we note that the result of all this should merely produce the factors, $A_{1 K}$ and $A_{2 K}$, as shown in the numerator of Equation (55). This will follow by the arguments given in the paragraph above Equation (48), where the expressions for a pair of oscillators are interpreted in terms of graphs with vertices and $\phi$-bonds. In this respect, the pair of half-planes can be regarded as a generalization of a pair of point particles. 


\section{B. Remark on a Formal Relationship to Quantum Field Theory}

As we stated above, the Fourier transform, $\tilde{\phi}(\omega)$, of the response function, $\phi(t)$, is the same as the Fourier transform, $\tilde{g}(K)$, of the correlation function, $g(K)$, at imaginary time, $\lambda=i t / \hbar$. The equality reads, Equation (15), being here reproduced for convenience:

$$
\tilde{\phi}(\omega)=\tilde{g}(K)
$$

where $K=i \hbar \omega$ is the imaginary frequency.

It turns out that the quantum statistical mechanics for particles and the quantum theory for fields are closely related, although the correspondence is not always so easy to see from a mere inspection. Therefore, we found it useful to point out how a parallel to Equation (B.1) reads in the conventional QFTfor the electromagnetic field.

Consider, for definiteness, Schwinger's source theory in the form presented, for instance, in [31]. For simplicity, as common in field theory, we work with natural units, so that $\hbar=c=k_{B}=1$. The electric field components, $E_{i}(x)$, are related to the polarization components, $P_{k}\left(x^{\prime}\right)$, via a tensor, $\Gamma_{i k}$, called the generalized susceptibility:

$$
E_{i}(x)=\int d^{4} x^{\prime} \Gamma_{i k}\left(x, x^{\prime}\right) P_{k}\left(x^{\prime}\right)
$$

where $x=(\mathbf{r}, t)$. Stationarity of the system means that $\Gamma_{i k}$ depends on time only through the difference, $\tau=t-t^{\prime}$. Causality implies that the integration over $t^{\prime}$ is limited to $t^{\prime} \leq t$.

Introduce the Fourier transform, $\Gamma_{i k}\left(\mathbf{r}, \mathbf{r}^{\prime}, \omega\right)$, via:

$$
\Gamma_{i k}\left(x, x^{\prime}\right)=\int_{-\infty}^{\infty} \frac{d \omega}{2 \pi} e^{-i \omega \tau} \Gamma_{i k}\left(\mathbf{r}, \mathbf{r}^{\prime}, \omega\right)
$$

The function, $\Gamma_{i k}\left(\mathbf{r}, \mathbf{r}^{\prime}, \omega\right)$, is known to be one-valued in the upper half frequency plane; it has no singularity on the real axis (omitting metals), and it does not take real values at any finite point in the upper half plane, except on the imaginary axis.

From Kubo's formula, we can now write:

$$
\Gamma_{i k}\left(\mathbf{r}, \mathbf{r}^{\prime}, \omega\right)=i \int_{0}^{\infty} d \tau e^{i \omega \tau}\left\langle\left[E_{i}(x), E_{k}\left(x^{\prime}\right)\right]\right\rangle
$$

This means that the generalized susceptibility can be identified with the retarded Green function: $\Gamma_{i k}\left(x, x^{\prime}\right)=G_{i k}^{R}\left(x, x^{\prime}\right)$. For $t<t^{\prime}$, both $\Gamma_{i k}\left(x, x^{\prime}\right)$ and $G_{i k}^{R}\left(x, x^{\prime}\right)$ vanish; the first because of causality, and the second because of the definition of the retarded Green function. [Note, as commented below, Equation (65), the Fourier transform in this section corresponds to the other convention, $\zeta=-i \omega$.]

Consider now the the correlation, $\left\langle E_{i}(x) E_{k}\left(x^{\prime}\right)\right\rangle$. Its Fourier transform, $\left\langle E_{i}(\mathbf{r}, \omega) E_{k}\left(\mathbf{r}^{\prime}, \omega^{\prime}\right)\right\rangle$ (in field theory, commonly called the two-point function), can be expressed in terms of the spectral correlation, $\left\langle E_{i}(\mathbf{r}) E_{k}\left(\mathbf{r}^{\prime}\right)\right\rangle_{\omega}$, as:

$$
\left\langle E_{i}(\mathbf{r}, \omega) E_{k}\left(\mathbf{r}^{\prime}, \omega^{\prime}\right)\right\rangle=2 \pi\left\langle E_{i}(\mathbf{r}) E_{k}\left(\mathbf{r}^{\prime}\right)\right\rangle_{\omega} \delta\left(\omega+\omega^{\prime}\right)
$$

Now, make use of the fluctuation-dissipation theorem [32] to get:

$$
\Im G_{i k}^{R}\left(\mathbf{r}, \mathbf{r}^{\prime}, \omega\right) \operatorname{coth}\left(\frac{1}{2} \beta \omega\right)=\left\langle E_{i}(\mathbf{r}) E_{k}\left(\mathbf{r}^{\prime}\right)\right\rangle_{\omega}
$$


with $\beta=1 / T$. Equation (B.6) is the field-theoretical counterpart of Equation (B.1). In both cases, we see that there is a close relationship between the response (or Green function) and the correlation.

To make the connection to the statistical mechanical method more explicit, one may consider expressions (B.3)-(B.6) for an oscillator, which has a frequency spectrum. Its response function (6) is:

$$
\phi(t)=\frac{1}{i \hbar} \operatorname{Tr}\{\rho[\mathrm{s}(0), \mathrm{s}(\mathrm{t})]\}
$$

where $s(t)$ is polarization or amplitude. The $\phi(t)$ corresponds to the $\Gamma_{i k}\left(x, x^{\prime}\right)$ of Equation (B.3), while the right-hand side of Equation (B.7) corresponds to the integrand of (B.4). Further, the Fourier transform, $\tilde{\phi}(\omega)$, corresponds to $\Gamma_{i k}\left(\mathbf{r}, \mathbf{r}^{\prime}, \omega\right)$ with $\omega \rightarrow-\omega$, due to definition (16) of $\tilde{\phi}(\omega)$.

Now, a simple harmonic oscillator expression (22) for $\tilde{g}_{a}(K) \rightarrow \tilde{g}(K) \rightarrow \tilde{\phi}(\omega)$ is valid. The inverse transform (17) to imaginary time, $\lambda=i t / \hbar$, will for this $\tilde{g}(K)$ give $\left(\omega_{a} \rightarrow \omega_{0}\right)$ :

$$
\begin{gathered}
g(\lambda)=\langle s(t) s(0)\rangle_{\omega_{0}}=\frac{1}{2} \alpha \hbar \omega_{0} \frac{\cosh \left(\frac{1}{2} \beta \hbar \omega_{0}-\lambda\right)}{\sinh \left(\frac{1}{2} \beta \hbar \omega_{0}\right)} \\
g(0)=\langle s(0) s(0)\rangle_{\omega_{0}}=\frac{1}{2} \alpha \hbar \omega_{0} \operatorname{coth}\left(\frac{1}{2} \beta \hbar \omega_{0}\right)
\end{gathered}
$$

with a distribution of eigenfrequencies, Equations (27) and (29) are valid. Therefore, from Equations (28) and (29), one finds $\left(m=\hbar \omega_{0}\right)$ :

$$
g(0)=\frac{1}{2 \pi} \int_{-\infty}^{\infty}\langle s(0) s(0)\rangle_{\omega_{0}} d \omega_{0}=\int_{0}^{\infty} \alpha_{I}\left(m^{2}\right) \frac{1}{2} m \operatorname{coth}\left(\frac{1}{2} \beta m\right) d\left(m^{2}\right)
$$

where with $K=i \hbar \omega_{0}$ :

$$
\alpha_{I}\left(m^{2}\right) m^{2}=-\frac{1}{\pi} \Im[g(K)]=-\frac{1}{\pi} \Im\left[\phi\left(\omega_{0}\right)\right]
$$

Now:

$$
\int_{0}^{\infty} m d\left(m^{2}\right)=2 \int_{0}^{\infty} m^{2} d m=\int_{-\infty}^{\infty} m^{2} d m
$$

so:

$$
g(0)=-\frac{1}{2 \pi} \int_{0}^{\infty} \Im\left[\phi\left(\omega_{0}\right)\right] \operatorname{coth}\left(\frac{1}{2} \beta m\right) d m
$$

Thus, with Equations (B.10) and (B.13), one finds $\left(m=\omega_{0}, \hbar=1\right)$ :

$$
-\Im\left[\phi\left(\omega_{0}\right)\right] \hbar \operatorname{coth}\left(\frac{1}{2} \beta m\right)=\langle s(0) s(0)\rangle_{\omega_{0}}
$$

which corresponds to Equation (B.6) of field theory. (The minus sign is due to the shift, $\omega \rightarrow-\omega$ by Fourier transform.)

However, by the statistical mechanical approach, Equation (B.6) is not of primary interest. Instead, relation (B.1), which is independent of temperature for harmonic oscillators, is crucial. Further, the electromagnetic field, which is quantized as a set of harmonic oscillators, can be eliminated to be replaced by its Green function, $\phi(\omega)=g(K)$, that acts as a pair interaction between induced dipole moments. 


\section{References}

1. Teodorovich, E.V. On the contribution of macroscopic van der Waals interactions to frictional force. Proc. R. Soc. Lond. A 1978, 362, 71-77.

2. Pendry, J.B. Shearing the vacuum-quantum friction. J. Phys.: Condens. Matter 1997, 9, 10301-10320.

3. Pendry, J.B. Can sheared surfaces omit light? J. Mod. Opt. 1998, 45, 2389-2408.

4. Pendry, J.B. Quantum friction-fact or fiction? New J. Phys. 2010, 12, 033028.

5. Volokitin, A.I.; Persson, B.N.J. Theory of friction: The contribution from fluctuating electromagnetic field. J. Phys.: Condens. Matter 1999, 11, 345.

6. Volokitin, A.I.; Persson, B.N.J. Noncontact friction between nanostructures. Phys. Rev. B 2003, 68,155420 .

7. Volokitin, A.I.; Persson, B.N.J. Near-field radiative heat transfer and noncontact friction. Rev. Mod. Phys. 2007, 79, 1291-1329.

8. Volokitin, A.I.; Persson, B.N.J. Theory of the interaction forces and the radiative heat transfer between moving bodies. Phys. Rev. B 2008, 78, 155437.

9. Volokitin, A.I.; Persson, B.N.J. Quantum friction. Phys. Rev. Lett. 2011, 106, 094502.

10. Dedkov, G.V.; Kyasov, A.A. Vacuum attraction, friction and heating of nanoparticles moving nearby a heated surface. J. Phys.: Condens. Matter 2008, 20, 354006.

11. Dedkov, G.V.; Kyasov, A.A. Conservative-dissipative forces and heating mediated by fluctuation electromagnetic field: Two plates in relative nonrelativistic motion. Surf. Sci. 2010, 604, 562-567.

12. Dedkov, G.V.; Kyasov, A.A. Dynamical van der Waals atom-surface interaction. Surf. Sci. 2011, 605, 1077-1081.

13. Dedkov, G.V.; Kyasov, A.A. Dynamical Casimir-Polder atom-surface interaction. Surf. Sci. 2012, $606,46-52$.

14. Philbin, T.G.; Leonhardt, U. No quantum friction between uniformly moving plates. New J. Phys. 2009, 11, 033035.

15. Høye, J.S.; Brevik, I. Casimir friction force and energy dissipation for moving harmonic oscillators. EPL 2010, 91, 60003.

16. Høye, J.S.; Brevik, I. Casimir friction force and energy dissipation for moving harmonic oscillators. II. Eur. Phys. J. D 2011, 61, 335-339.

17. Høye, J.S.; Brevik, I. Casimir friction in terms of moving harmonic oscillators: Equivalence between two different formulations. Eur. Phys. J. D 2011 64, 1-3.

18. Høye, J.S.; Brevik, I. Casimir friction force between polarizable media. Eur. Phys. J. D 2012 , $66,149$.

19. Høye, J.S.; Brevik, I. Casimir friction force for moving harmonic oscillators. Int. J. Mod. Phys. A 2012, 27, 1260011.

20. Høye, J.S.; Brevik, I. Friction force between moving harmonic iscillators. Physica A 1992, 181, 413-426.

21. Brevik, I.; Høye, J.S. Van der Waals force derived from a quantum statistical mechanical path integral method. Physica A 1988, 153, 420-440. 
22. Barton, G. On van der Waals friction: I. Between two atoms. New J. Phys. 2010, 12, 113044.

23. Barton, G. On van der Waals friction. II: Between atom and half-space. New J. Phys. 2010, $12,113045$.

24. Barton, G. On van der Waals friction between two atoms at nonzero temperature. New J. Phys. 2011, 13, 043023.

25. Kubo, R. Lectures in Theoretical Physics (Lectures delivered at the Summer Institute for Theoretical Physics, University of Colorado, Boulder, 1958); Brittin, W.E., Dunham, L.G., Eds.; Interscience: New York, NY, USA, 1959; Volume I.

26. Høye, J.S.; Olaussen, K. Eigenmodes of the quantized polarizable fluid. J. Chem. Phys. 1982, $77,2583$.

27. Høye, J.S.; Brevik, I. Van der Waals force between dielectric plates derived from the quantum statistical mechanical path integral method. Physica A 1998 259, 165-182.

28. Høye, J.S. Casimir Force for Electrolytes. In The Casimir Effect and Cosmology; Odintsov, S.D., Elizalde, E., Gorbunova, O. G., Eds.; Tomsk State Pedagogical University: Tomsk, Russia, 2008; pp. 117-124; e-print arXiv:0903.2975.

29. Høye, J.S.; Brevik, I. Casimir force between dielectric media with free charges. Phys. Rev. E 2009, $80,011104$.

30. Høye, J.S.; Stell, G. Statistical mechanics of polar systems. II. J. Chem. Phys. 1976, 64, 1952.

31. Schwinger, J.; DeRaad, L.L., Jr.; Milton, K.A. Casimir effect in dielectrics. Ann. Phys. (N.Y.) 1978, $115,1-23$.

32. Landau, L.D.; Lifshitz, E.M. Statistical Physics, 3rd ed.; Pergamon Press: Oxford, UK, 1980; Part 1.

(C) 2013 by the authors; licensee MDPI, Basel, Switzerland. This article is an open access article distributed under the terms and conditions of the Creative Commons Attribution license (http://creativecommons.org/licenses/by/3.0/). 